\title{
Spectroscopy and Remote Sensing Techniques to Assess Active- and Post-Fire Effects ${ }^{+}$
}

\author{
Anna Brook \\ The Spectroscopy and Remote Sensing Laroratory, University of Haifa, Abba Khoushy Ave 199, Haifa 3498838, \\ Israel; abrook@geo.haifa.ac.il \\ + Presented at TERRAenVISION 2019, Barcelona, 2-7 September 2019.
}

Published: 29 May 2020

\begin{abstract}
Fires were once a natural phenomenon that helped to shape species distribution, contributed to the persistence of fire-dependent species, and assisted the natural evolution of ecosystems. However, nowadays, most of the forest fires worldwide are not of natural causes. Therefore, wildfires have received significant attention over the past few decades. Major ecological and policy changes were stimulated by historical frequency, extent, and severity of fires in the dry forests. These fires are important at both local to regional scales, as it might change the maintenance of landscape structure, composition, and function. Moreover, it affects pollutants, impacts air quality and raises human health risks. Many studies suggested using remote sensing data and techniques to assess fire characteristics and post-fire effects. Due to its ability to quantify patterns of variation in space and time, the remote sensing data are especially important to detect active fire extents at local and regional scales, mapping fuel loading and identify areas with long or problematic natural recovery. In the past few decades, the advantages of multi-temporal remote sensing techniques to monitor landscape change in a rapid and cost-effective manner, are reported in the scientific literature. Many studies focused on the development of techniques to evaluate and quantify fire behavior and fuel combustion. Yet the main contribution is recorded for spectral indices, e.g. the Normalized Burn Ratio (NBR), the difference in the Normalized Burn Ratio between pre- and post-fire images (dNBR), and the Normalized Difference Vegetation Index (NDVI), which are calculated by a simple combinations of different sensor bands, rely on spectral changes of the burning or burned surfaces. Numerous papers are focused on more advanced and very detailed spectral models of fuel and post-fire ash residues, mainly using laboratory spectrometers, e.g., Fourier Transform Infrared (FTIR). However, many of the developed models are not applicable in the real world. In the current talk, we will present the most recent studies and scientific activities in the field of (1) active fire detection and characterization, using mainly hyperspectral ground and airborne technologies; (2) future space-borne applications on board of nano- and micro-satellites; (3) discuss the contribution of detailed and precise spectral models for post-fire ecological effects studies; (4) describe field assessment; (5) discuss management applications and future directions of fire-related remote sensing research.
\end{abstract}

Keywords: spectroscopy; remote sensing; wildfire; pollution; ash 